\title{
The Impact Analysis of High Speed Railway on City Accessibility in Henan Province
}

\author{
Kaiguang Zhang ${ }^{*}$, Mingting Ba and Hongling Meng \\ Institute of 3S Technology, Zhengzhou Normal University, Zhengzhou 450044, China \\ ${ }^{*}$ Corresponding author
}

\begin{abstract}
Accessibility level is an important indicator to measure the perfect degree of traffic network, *-shaped high-speed railway network in Henan province, forming in the next 10 years, will have significant impacts on the accessibility spatial distribution pattern. In the paper, those impact and the evolution patterns, in 2010, 2016, 2020 and 2025, were analyzed by using the time distance between cities and population weighted travel time inverse as accessibility indexes. The results show that the construction and improvement of high speed railway traffic network, will effectively improve the links among cities, shorten the distance between them, result an obvious space convergence effect. By 2025, as ZhengJi and ZhengTai high speed railways opening to traffic, one hour of high speed railway living area with Zhengzhou as the center will have been formed in the province. City accessibility indexes exhibit center-edge characteristics, the accessibility levels of cities in central region are significantly higher than that of cities in marginal region. Different high speed railway lines have significant differences on the promotion of province accessibility levels, ZhengTai and ZhengJi high speed railway lines have the largest contribution rate per $100 \mathrm{~km}$, followed by JingGuang and XuLan lines, ZhengWan and ZhengHe lines. The province accessibility contours gradually show slender spindle distribution characteristics with Zhengzhou as center, JingGuang and XuLan high speed railway lines as major and minor axes respectively, contour curves tend to be smooth and uniform. The province accessibility will have been optimizing, and the distribution of railway network will have been becoming space equalization.
\end{abstract}

Keywords-high speed railway; time distance; city accessibility; logistics curve; space interpolation

\section{INTRODUCTION}

Since the first railway was opened to traffic in 1876, Chinese railway has gone through 140 years of railway construction process. Railway for its safe, fast and low cost has become a major revolution in human transportation history. By the end of 2009, WuGuang high-speed railway official operation marks the China officially entered the era of highspeed railway, The development of high speed railway network is promoting the rapid growth of the national transportation hub city, the dense area and the traffic economic belt, and the expansion of the urban system. With the maturity of high-speed rail technology, the regional spatial geographic conditions and economic development plays an important role in the formation of high speed railway traffic network. A reasonable high-speed railway network can not only greatly improve the regional transport capacity, can also provide a relying axis for the regional economic development.
An important evaluating indicator of regional traffic network is the network accessibility level, which describes the interacting degree, transport ease degree between nodes in the traffic network[1-3]. Domestic researches on accessibility started in the late 1980s, the current researches mainly focused on the accessibility spatial distributions, the spatial distribution characteristics, the evolution patterns of a traffic network, and its impact on regional development within a specific region. But the forecast study for the future phases of network construction is very few [4-14].

Henan Province, located in the middle of China, is one of the most important transport hub and logistics centers in the country. By the end of 2016, along with the construction of ZhengWan and ZhengHe high speed railway lines, and the preconstruction of ZhengTai and ZhengJi high speed railway lines, the *-shaped high-speed railway network with Zhengzhou as the center will have been forming in the near future(Figure 1).

In this study, the distribution characteristics of city accessibilities, the future impact of the phased high speed railway constructions are analyzed by using spatial analysis methods, in order to find the weaknesses of the provincial traffic network in the high speed railway era, provide useful references for making the coordinated regional development policy in the province.

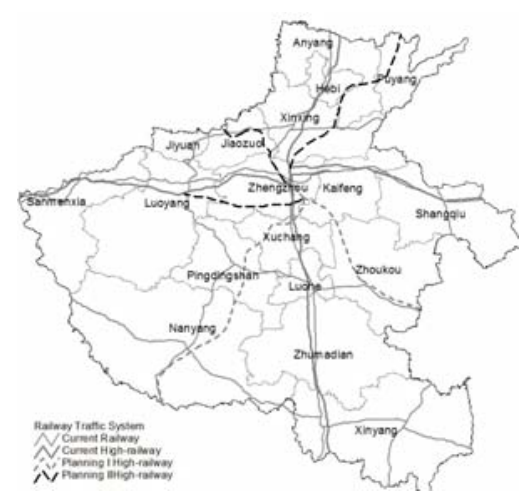

FIGURE I. RAILWAY NETWORK SYSTEM IN HENAN

For facilitating vertical comparison, calculating the impact of high speed railway construction on the city accessibility, referring Zhengzhou Modern Integrated Transport Hub Development Plan, the study divides the formation process of Henan high speed railway network into four time sections and three time periods, respectively are the first time section (2010) on which the province run into the high-speed railway era, the 
second time section (2016) the status quo year, the third time section (2020) on which ZhengWan and ZhengHe lines will officially open to traffic, the forth time section (2025) on which ZhengJi and ZhengTai lines will officially open to traffic.

\section{RESEARCH METHODS}

Accessibility describes the interacting degree, transport ease degree between nodes in the traffic network. This paper analyzes the impact of Henan high speed railway network constructions on the accessibility distributions characteristics and their development patterns in the view of the city spatial relations and regional population distribution.

\section{A. Space Distance}

The space distance between cities is a basis indicator to measure network accessibility level, the space distance $\boldsymbol{D}_{\boldsymbol{i j}}$ between $\boldsymbol{i}$ and $\boldsymbol{j}$ is defined as the length of the shortest space distance route in the network, is calculated recursively by the space distance between the adjacent cities $D_{i j}=\sum_{k \in R(D) i} D_{i k}^{0}$. The city $\boldsymbol{i}$ space distance radius $D_{i}$ is defined as the sum of the space distances between $\boldsymbol{i}$ and other cities in the network,

$$
D_{i}=\sum D_{i j}
$$

which describes the space location of $i$ in the network, the smaller the closer to the center of the network.

The network space distance radius $\boldsymbol{D}$ is defined as the half of the sum of city space distance radiuses

$$
D=\frac{1}{2} \sum D_{i}
$$

The smaller $\boldsymbol{D}$ the network spatial distribution is more balanced.

\section{B. Network Running Speed}

To accurately describe the impact of high-speed railway construction on the efficiency of network operation, the speed of railway running at different time sections should be set. On the first and second time sections, the railway running speeds are set as the weighted averages of train running speeds in the corresponding year railway running map, respectively are, ordinary railway $140 \mathrm{~km} / \mathrm{h}$, ordinary railway $150 \mathrm{~km} / \mathrm{h}$ and high-speed railway $250 \mathrm{~km} / \mathrm{h}$. Referring to National Railway Long-term Planning and the empirical data of the 6 times speed increase in China, on the third andforth time sections, the railway running speeds are respectively set as $160 \mathrm{~km} / \mathrm{h}$ and $285 \mathrm{~km} / \mathrm{h}, 170 \mathrm{~km} / \mathrm{h}$ and $320 \mathrm{~km} / \mathrm{h}$.

\section{Time Distance}

The time distance between cities in the network, is an important indicator to measure the network transport ease, the time distance $\boldsymbol{R}(\boldsymbol{T})_{i j}$ between city $\boldsymbol{i}$ and $\boldsymbol{j}$ is defined as the time consumption of the shortest time route in the network, is calculated recursively by the time consumption between the adjacent cities $T_{i j}=\sum_{k \in R\left(T_{i j}\right)_{i k}^{0}}$. The city $i$ time distance radius $\boldsymbol{T}_{i}$ is defined as the sum of the time distances between $i$ and other cities in the network,

$$
T_{i}=\sum T_{i j},
$$

which reflects the network traffic convenience degree of $i$, the smaller the more developed in the network, the higher the convenience degree.

The network time distance radius $\boldsymbol{T}$ is defined as the half of the sum of city time distance radiuses

$$
T=\frac{1}{2} \sum T_{i}
$$

which reflects the network operating level, the smaller $\boldsymbol{T}$ the higher the efficiency of the entire network.

Space distance and time distance, reflect different aspects of the spatial distribution characteristics of a traffic network, there are some differences between them. Due to the shortest time route is not the shortest space distance route $\left(\boldsymbol{R}(\boldsymbol{T})_{i j} \neq \boldsymbol{R}(\boldsymbol{D})_{i j}\right)$, sometimes there are $\boldsymbol{D}_{i}>\boldsymbol{D}_{j}$, but $\boldsymbol{T}_{i}<\boldsymbol{T}_{j}$ [15].

\section{Accessibility Index}

The purpose of traffic network construction and improvement is to enhance logistics and passenger transport capacity, and better serves the social and economic development. Reasonable accessibility index reflects not only the transport network structure and spatial distribution, but also has the characteristics of population spatial distribution in the study area [16].

City accessibility index $A_{i}$ is defined as the weighted average of the inverse of the shortest time distance $\boldsymbol{t}_{i j}$ from the city to all other cities in the traffic network system

$$
A_{i}=\sum_{j \neq i} \frac{M_{j}}{t_{i j} \sum_{k \neq i} M_{k}}
$$

where, the use of the inverse is to effectively reduce the impact of numerical attributes on the analysis results, and facilitate the multiple equivalent path problems between two cities in traffic network, $\boldsymbol{M}_{\boldsymbol{j}}$ is the influencing degree of $\boldsymbol{j}$ on $\boldsymbol{i}$, use the resident population of $\boldsymbol{j}$ in the paper.

The larger the accessibility $\boldsymbol{A}_{\boldsymbol{i}}$, the greater the traffic efficiency of $i$ with all other cities in the region.

The network accessibility $\boldsymbol{A}$ is defined as the sum of city accessibility of all cities in the network,

$$
A=\sum A_{i},
$$

The larger $\boldsymbol{A}$ the better accessibility of the network, the higher the efficiency of the regional population circulation. its variation over time reflects the sophistication of network development. 


\section{E. Resident Population Forecast Model}

In order to accurately estimate the impact of population changes on city accessibility, the number of resident population for all cities on future time sections need to be forecasted.

On the first and second time sections, the resident population data come from corresponding Henan Statistical Yearbook. On the third and forth time sections, the resident population data are forecasted by using logistics curve model and the resident population data in Henan Statistical Yearbook (2012-2016).

The calculation formulas are

$$
\frac{d N}{d t}=r N\left(1-\frac{N}{K}\right), N=\frac{K}{1+\exp \{a-r t\}},
$$

where $N$ is the number of resident population, $t$ is the time series, the constant $\boldsymbol{r}$ indicates the natural growth rate, $\boldsymbol{\kappa}$ is the environment load force.

$$
K=\frac{N_{1} N_{4}\left(N_{2}+N_{3}\right)-N_{2} N_{3}\left(N_{1}+N_{4}\right)}{N_{1} N_{4}-N_{2} N_{3}}, \quad, \quad a, r \text { is the regression }
$$

coefficients of $y=\ln \frac{K-N}{N}=a-r t$.

\section{The IMPACt ANALysis OF High SPEED RaIlWAY CONSTRUCTION ON CITY ACCESSIBILITY}

\section{A. Spatial Distribution Characteristics Evolution Pattern}

Using (1)-(4) to calculate the space distance radiuses and the time distance radiuses of the province and all cities on the four time sections, the results are shown in Table 1.

\begin{tabular}{|c|c|c|c|c|c|c|c|c|c|c|c|c|c|c|c|c|c|c|c|}
\hline & ZZ & KF & LY & PDS & AY & HB & $\mathbf{X X}$ & $\mathbf{J Z}$ & PY & XC & LH & SMX & NY & SQ & XY & ZK & PDS & JY & HN \\
\hline 10TD & 22 & 29.2 & 28.6 & 29.1 & 35.7 & 311 & 255 & 30.2 & 34.7 & 245 & 26.1 & 43.4 & 426 & 44.2 & 43.2 & 34.1 & 33.4 & 31.4 & 294.3 \\
\hline 16TD & 14.1 & 17.6 & 18.6 & 21.5 & 21.6 & 18.4 & 15.5 & 21.9 & 22 & 15.6 & 17.1 & 27.1 & 33.8 & 26.4 & 27.6 & 24.3 & 20.9 & 23.8 & 193.9 \\
\hline 20TD & 11 & 14 & 16 & 15.8 & 17.5 & 14.9 & 12.2 & 19.3 & 18.4 & 13.7 & 15.1 & 22.7 & 22.6 & 21.2 & 24.6 & 17.2 & 18.8 & 21.8 & 158.4 \\
\hline 25TD & 8.83 & 11.5 & 14.3 & 13.9 & 15.7 & 13.4 & 11 & 12.4 & 15.8 & 11.5 & 12.5 & 20.2 & 19.9 & 18 & 21.3 & 14.8 & 15.9 & 16.2 & 133.4 \\
\hline 10SD & 3.08 & 4.09 & 4.00 & 4.07 & 4.97 & 4.36 & 3.56 & 4.23 & 4.82 & 3.42 & 3.65 & 6.07 & 5.96 & 6.18 & 6.05 & 4.77 & 4.67 & 4.40 & 41.17 \\
\hline 16SD & 2.91 & 3.81 & 4.00 & 3.95 & 4.86 & 4.20 & 3.43 & 3.52 & 4.61 & 3.27 & 3.62 & 5.92 & 5.73 & 5.88 & 5.92 & 4.55 & 4.55 & 4.04 & 39.37 \\
\hline 20SD & 2.71 & 3.62 & 3.95 & 3.54 & 4.67 & 4.01 & 3.24 & 3.47 & 4.61 & 3.27 & 3.62 & 5.92 & 5.26 & 5.69 & 5.92 & 4.11 & 4.55 & 3.99 & 38.07 \\
\hline 20SD & 2.71 & 3.61 & 3.95 & 3.54 & 4.67 & 4.01 & 3.24 & 3.47 & 4.58 & 3.27 & 3.62 & 5.92 & 5.26 & 5.69 & 5.92 & 4.10 & 4.55 & 3.99 & 38.04 \\
\hline
\end{tabular}

TABLE I. THE TIME AND SPACE RADIUSES OF CITIES IN HENAN
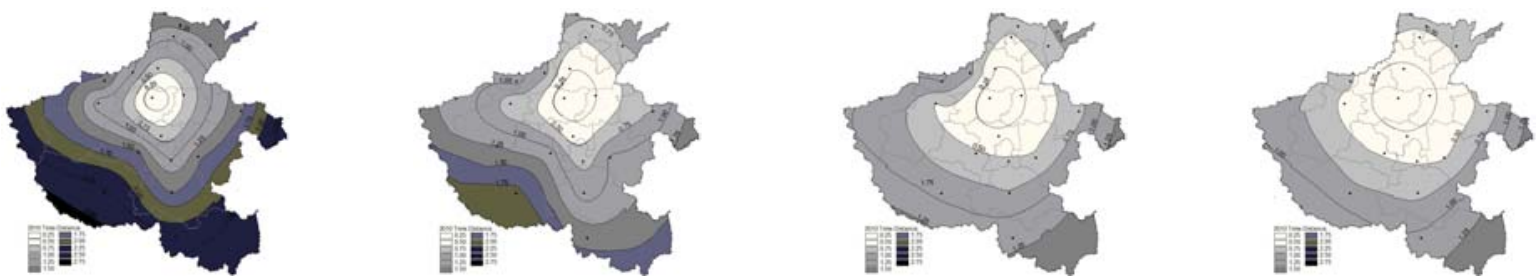

FIGURE II. INTERPOLATION ANALYSIS OF TIME DISTANCE IN HENAN

On the four time sections, although the ordering of city space radiuses are different, the shortest three cities (Zhengzhou, Xinxiang and Xuchang) and The longest three cities remain unchanged, These means that city space radius is closely related to its space location, cities closer to the network center have smaller space radiuses.

The time distance is based on the space distance, at the same time consider train running speed. The high speed railway shortens the time distances between cities along the railway lines, makes the ordering of city space distance and city time distance are evidently different on the four time sections, but the general trend is consistent. The shortest three cities always are Zhengzhou, Xinxiang and Xuchang. The longest three cities always are Shangqiu, Sanmenxia and Xiyang. Compared to the previous time section, on the second time section, the improvement degree of city time distance radiuses is between $20.75 \%-40.84 \%$, of which the best three cities respectively are Heb, Shangqiu and Kaifeng, decrease respectively $40.84 \%, 40.16 \%$ and 39.78 , the poorest three cities respectively are Nanyang, Jiyuan and Pingdingshan, decrease respectively $20.75 \%, 24.29 \%$ and $26.29 \%$. On the third time section, the improvement degree of city time distance radiuses is between $8.49 \%-33.2 \%$, of which the best three cities respectively will be Nanyang, Zhoukou and Pingdingshan, decrease respectively $33.20 \%, 29.14 \%$ and $26.15 \%$, the poorest three cities respectively will be Jiyuan and Zhumadian and Xinyang, decrease respectively $8.49 \%$ 、 $10.36 \%$ and $10.70 \%$. On the forth time section, the improvement degree of city time distance radiuses is between $19.47 \%-35.87 \%$, of which the best three cities respectively will be Jiaozuo, Jiyan and Zhengzhou, decrease respectively $35.87 \%$ 、 $25.67 \%$ and $19.47 \%$, the poorest three cities respectively will be Xinxiang, Anyang and Hebi , decrease respectively $10.19 \%$ 、 $10.22 \%$ and $10.22 \%$. 
The construction of high-speed railway has greatly shortened the time distance between cities, making the average time distance between the cities in the province from $1.92 \mathrm{~h}$ in 2010, shortened to $0.87 \mathrm{~h}$ in 2025 . For Zhengzhou space distance radius and time distance radius are minimal on the four time sections, the evolution patterns of city time distances in the province can be obtained by spatial interpolation of its time distance distributions (Figure 2).

In 2010, Zhengzhou time distance radius and space distance radius are basically similar, showing a uniform change characteristic. by 2016, as the opening of JingGuang and Xulan high speed railway, the contours show elliptically symmetric distribution characteristics, with JiingGuang and Xulan high speed railway lines as the major axis and the minor axis, the impact of JiingGuang and Xulan high speed railway lines are significantly higher than that of Xulan high speed railway lines.

By 2020, the opening of ZhengWan and ZhengHe high speed railways will shorten the time distance between Zhengzhou and the cities in the southwest and southeast pats of the province, the time contours clearly shifts to the two directions. The contours in the south of XuLan high speed railway line show some circular distribution characteristics, the changes of the contours and the space distances tends to be consistent.

By 2025, the opening of ZhengTai and ZhengJi high speed railways will change the contours triangular distribution characteristics in the north of XuLan high speed railway line, The contours show some circular distribution characteristics, the changes of the contours and the space distances are consistent, one hour high speed railway life circle will have been basically formed.

\section{B. Accessibility Distribution Characteristics Evolution Pattern}

The resident population data on the next 2 time sections are forecasted by using Logistics curve model and the resident population data in Henan Statistical Yearbook(2012-2016). The province and city accessibilities are calculated on the 4 time sections by using (5) and (6), the results are showed in Table 2 and Figure 3 The spatial interpolation analysis results are showed in the Figure 4.

TABLE II. THE CITY ACCESSIBILITIES OF 4 TIME SECTION IN HENAN

\begin{tabular}{|c|c|c|c|c|c|c|c|c|c|c|c|c|c|c|c|c|c|c|c|}
\hline & $\mathbf{Z Z}$ & KF & $\mathbf{L Y}$ & PDS & AY & HB & XX & $\mathbf{J Z}$ & PY & XC & LH & SMX & NY & SQ & XY & ZK & PDS & JY & HN \\
\hline $10 \mathrm{AC}$ & 0.89 & 0.74 & 0.65 & 0.77 & 0.62 & 0.92 & 0.83 & 0.69 & 0.69 & 0.90 & 1.06 & 0.44 & 0.48 & 0.43 & 0.55 & 0.65 & 0.71 & 0.67 & 12.69 \\
\hline $16 \mathrm{AC}$ & 1.53 & 1.38 & 0.98 & 0.94 & 1.01 & 1.49 & 1.48 & 0.89 & 0.99 & 1.41 & 1.53 & 0.71 & 0.56 & 0.76 & 0.79 & 0.85 & 1.10 & 0.83 & 19.24 \\
\hline $20 \mathrm{AC}$ & 1.92 & 1.71 & 1.15 & 1.34 & 1.20 & 1.74 & 1.79 & 0.97 & 1.13 & 1.61 & 1.71 & 0.85 & 0.88 & 0.93 & 0.88 & 1.16 & 1.23 & 0.88 & 23.09 \\
\hline $20 \mathrm{AC}$ & 2.28 & 2.01 & 1.30 & 1.48 & 1.34 & 1.92 & 2.02 & 1.70 & 1.29 & 1.84 & 1.89 & 0.96 & 0.98 & 1.07 & 0.97 & 1.29 & 1.40 & 1.18 & 26.93 \\
\hline
\end{tabular}
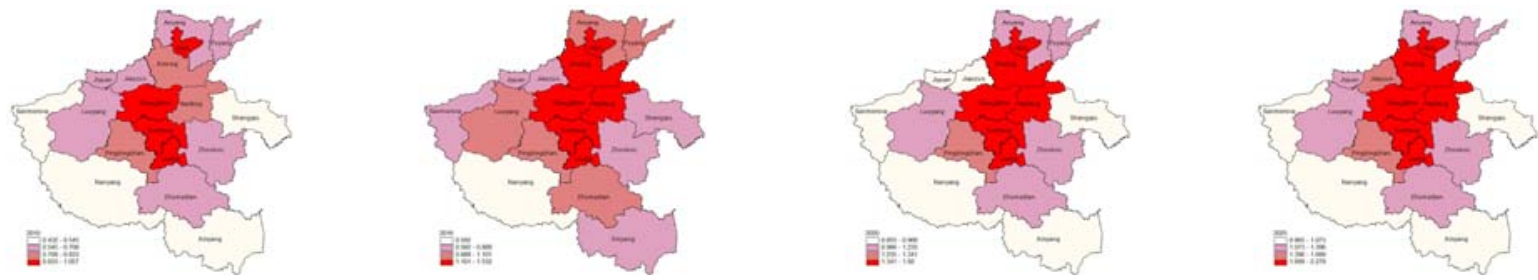

FIGURE III. SPATIAL DISTRIBUTION OF CITY ACCESSIBILITIES IN HENAN
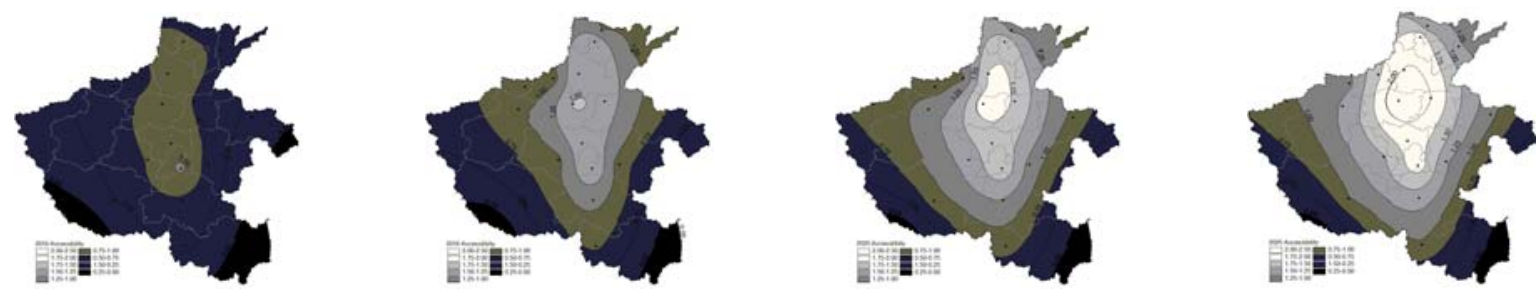

FIGURE IV. INTERPOLATION ANALYSIS OF CITY ACCESSIBILITIES IN HENAN

The opening of high-speed railway will have been effectively improved the province accessibility level, in the 3 time periods, the province accessibility levels increase respectively by $51.64 \%$, $20 \%$ and $16.61 \%$.

The largest contribution rate per $100 \mathrm{~km}$ is Zhengtai and ZhengJI high speed railway lines in the third time period, up to $6.34 \%$, followed by JingGuang and XuLan lines in the first time period, which link 12 cities of 18 cities, and covering about $70 \%$ of the province's population, then ZhengWan and ZhengHe lines in the second time period.

The opening of high-speed railway also effectively improve the accessibility levels of the cities along the railway lines, In the first time period, the best three cities are Kaifeng, Shangqiu and Xinxiang, increase respectively 40.84\%, 40.16\% and 39.78. In the second period, the best three cities will be Nanyang, Pingdingshan and Zhoukou, increase respectively 
$57.27 \%, 72.73 \%$ and $36.10 \%$. In the third period, the best three cities will be Jiaozuo, Jiyuan and Zhengzhou, increase respectively $75.91 \%$, 33.93\% and $18.70 \%$.

Overall, the construction of high speed railway will greatly improve the city accessibility levels. Affected by the distribution of geographical location and population, there are significant differences in the improvement degrees. The cities with maximum improvements are Shangqiu, Zhengzhou and Kaifeng, have $148.55 \%, 156.73 \%$ and $148.56 \%$ respectively. The cities with minimum improvements are Shangqiu, Zhengzhou and Kaifeng, have $75.12 \%, 78.46 \%$ and $79.14 \%$ respectively.

On the four time sections, the spatial distributions of city accessibilities have the center to edge characteristics, the accessibilities of cities in the central region are higher than that of cities in marginal regions. In part because the city accessibility is affected by city geographic location, but the main reason is the larger density of population, railway lines and cities in the central region.

Dividing the accessibility levels into excellent, good, fair, and poor four grades, the central cities, along JingGuang high speed railway line, as Hebi, Zhengzhou, Xuchang and Luohe are always in the excellent grade, the marginal cities such as Sanmenxia, Nanyang and Shangqiu are always in the poor grade on the four time sections. XuLan high speed railway and ZhengKai intercity railway effectively improve the accessibility of Kaifeng on the second time section, make it promote into the best city grade. In the 10 years from the second to the fourth time sections, the sequences of city accessibilities are basically unchanged.

The accessibility contours(Figure 4) gradually show the slender spindle distribution characteristics with major and minor axes respectively coincide with JingGuang high speed railway line and XuLan high speed railway line, Zhengzhou as the center. From 2010 to 2025, the accessibility contours tend to be smooth and uniform, as a whole will have been optimizing. These indicate that the construction of high-speed railway is of great significance to improve the regional city accessibility levels, the distribution of the province railway network will have been becoming space equalization.

\section{CONCLUSION}

The construction and improvement of high speed railway network, effectively improve the links among cities, shorten the distance between them, result an obvious space convergence effect. By 2025, with ZhengJi and ZhengTai high speed railways opening to traffic, one hour of high speed railway living area with Zhengzhou as the center will have been formed in the province.

City accessibility indexes show center-edge characteristics, the accessibility levels of cities in central region are significantly higher than that of cities in marginal regions. Different high speed railway lines have significant differences on the promotion of province accessibility levels, ZhengTai and ZhengJi high speed railway lines have the largest contribution rate per $100 \mathrm{~km}$, followed by JingGuang and XuLan lines, and ZhengWan and ZhengHe lines.
The province accessibility contours gradually show some slender spindle distribution characteristics with Zhengzhou as center, JingGuang and XuLan high speed railway lines as major and minor axes respectively, contour curves tend to be smooth and uniform. The province accessibility will have been optimizing, and the distribution of railway network will have been becoming space equalization.

\section{REFERENCES}

[1] J. Fengjun, W. Jiaoer, "Railway network expansion and spatial accessibility analysis in China:1906-2000,” Acta Geographica Sinica, vol. 59, pp. 293-302, February 2004.

[2] Z. Shaoying, G. Yebo. "The impact of accessibilityof railway network with high speed railway construction in China," Areal research and development, vol. 32, pp. 46-51, February 2013.

[3] D. Qiuxian, Z. Lixia, "Impact of high speed railway on accessibility and economic linkage of cities along the railway,” Journal of Central China Normal University(Natural Sciences), vol. 49, pp. 952-956, June 2015.

[4] L. Pinghua, L. Yulin, "Retrospect and prospect of accessibility research ,” Journal of geographical science, vol. 24, pp. 69-77, March 2005.

[5] Henrike R., Amaya V., "Spatial (Im) mobility and accessibility in Ireland: Implications for transport policy,” Growth and Change, vol. 43, pp. 667-696, April 2012.

[6] L. Qinghua, W. Xinyan , "Research on the public transportation accessibility in Lhasa,” Journal of Tibet University (Natural Science), vol. 28, pp. 117-120, January 2013.

[7] S. Jinghong, L. Yuqi, L. Xiaoji, "Assessment on accessibility of regional comprehensive transport: a case study of Anhui," Geographical Research, vol. 31, pp. 1280-1293, July 2012.

[8] X. Jingge, Z. Tinggang, "Spatial-temporal Characteristics of Chongqing Transport Network Accessibility and Its Evolution Pattern,” Journal of Geo-information sicience,vol. 17, pp. 54-61, January 2015,

[9] L. Baojie, G. Hehe, J. Yazhou, "Study on accessibility of transportation infrastructure in urban and rural area of Xuzhou based on GIS,” Human Geography, pp. 76-80, June 2012.

[10] J. Xiaowei, "Spatial pattern and evolution of road network accessibility in Anhui Province ,” Progress in Geography, vol. 31, pp. 1591-1599 , December 2012.

[11] C. Xiaoshu, X. Desheng, Y. XiaoPei, "A study on the Urban accessibility of national trunk highway system in China," Acta Geographica Sinica, vol. 60, pp. 903-910, June 2005.

[12] W. Wei, C. Youhui, "Spatial structure and evolution of highway accessibility in the Yangtze River Delta,” Acta Geographica Sinica, vol. 61, pp. 1065-1074, January 2006.

[13] Z. Li, L. Yuqi, "Assessment on regional accessibility based on land transportation network: a case study of Yangtze river delta," Acta Geographica Sinica, vol. 61, pp. 1235-1246, December 2006.

[14] Z. Bing, J. Fengjun, "The appraise of accessibility on the evolution of highway network in Hunan province,” Economic geography, vol. 26, pp. 776-779, May 2006.

[15] Z. Kaiguang, B. Mingting, "Study on spatial distribution characteristics of intra-city economic sector value-added growth rates in Henan province,” Advance in Engineering Research, vol. 23, pp. 578-583, May 2015.

[16] Z. Kaiguang, M. Hongling, B. Mingting, “The research on optimum algorithms in non-Euclidean distance space,” Journal of Geomatics Science and Technology, vol.30, pp. 83-86, January 2013.

[17] distribution,” Xianmen university,1990. 